\title{
Respuesta ovulatoria y embrionaria a la somatotropina bovina recombinante en cabras superovuladas con FSHp
}

\section{Ovulatory and embryonic response to recombinant bovine somatotropin in goats superovulated with FSHp}

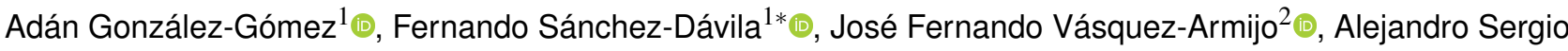

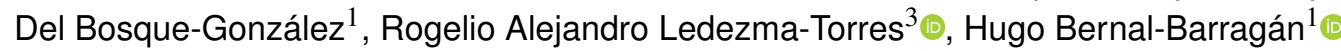 \\ ${ }^{1}$ Universidad Autónoma de Nuevo León, Facultad de Agronomía, Laboratorio de Reproducción Animal, Unidad Académica Marín, \\ Carretera Zuazua-Marín, km 17.5, CP. 66700. Marín, Nuevo León, México. \\ ${ }^{2}$ Universidad Autónoma del Estado de México, Centro Universitario UAEM Temascaltepec, Carretera Toluca-Tejupilco, km 67.5, CP. \\ 51300. Barrio de Santiago, Temascaltepec de González, Estado de México, México. \\ ${ }^{3}$ Universidad Autónoma de Nuevo León, Facultad de Medicina Veterinaria y Zootecnia, Av. Francisco Villa S/N Col. Ex Hacienda el \\ Canadá, CP. 66050. General Escobedo, Nuevo León, México. \\ *Autor de correspondencia: fernando_sd3@hotmail.com
}

Nota científica recibida: 25 de agosto de 2017 aceptado: 07 de septiembre de 2018

RESUMEN. El objetivo del estudio fue evaluar el efecto de la somatotropina bovina recombinante (rBST) en la actividad ovárica, cantidad y calidad de embriones transferibles en cabras durante el anestro estacional. Se utilizaron 30 cabras multíparas sincronizadas con protocolo corto de CIDR y superovuladas con FSHp, asignadas a tres tratamientos $(n=10)$ : $\mathrm{T} 1=$ control, $\sin \mathrm{rBST}, \mathrm{T} 2=100 \mathrm{mg}$ rBST y $\mathrm{T} 3=200 \mathrm{mg}$ rBST. Las variables cuantitativas se analizaron mediante prueba de Kruskal-Wallis; el porcentaje de estro y de embriones recuperados, mediante prueba de Chi - cuadrada. Las cabras del T3 tuvieron mayor número de $\mathrm{CL}$, seguidas del T2 $(p=0.002)$. El número de embriones recuperados y de blastocitos fue mayor en T3 $(p=0.001)$. El número de mórulas fue mayor en T1 $(p=0.001)$. La aplicación de rBST al inicio del estro incrementa la actividad ovárica y la cantidad de embriones transferibles en cabras.

Palabras clave: Cabras, embrión, protocolo corto, recolección de embriones, superovulación.

ABSTRACT. The objective of this study was to evaluate the effect of recombinant bovine somatotropin (rBST) on ovarian activity and quantity and quality of transferable embryos in goats during seasonal anoestrus. We used 30 multiparous goats synchronized with goats synchronized with CIDR short protocol and superovulated with FSHp, assigned to three treatments $(n=10): T 1=$ control, without rBST, T2 $=100 \mathrm{mg} \mathrm{rBST}$ y T3 $=200 \mathrm{mg} \mathrm{rBST}$. Quantitative variables were analyzed using the Kruskal-Wallis test, and the percentage of estrus and embryos recovered by the Chi-square test. A greater number of $\mathrm{CL}$ were registered in goats assigned to T3, followed by T2 $(p=0.002)$. The number of recovered embryos and blastocysts was higher in T3 $(p=0.001)$. The morula number was higher in T1 $(p=0.001)$. The application of rBST at the onset of estrus increases ovarian activity and the number of transferable embryos in goats.

Key words: Goats, embryo, short protocol, embryo collection, superovulation.

\section{INTRODUCCIÓN}

Para aumentar el número y calidad de embriones al comienzo de un programa de superovulación, se han diseñado y evaluado diversas estrategias, entre las cuales destacan: 1) El uso de agonistas $\mathrm{y} / \mathrm{o}$ antagonistas de la hormona liberadora de gonadotropinas $(\mathrm{GnRH})$ previos a la superovulación para incrementar el número de folículos pequeños disponibles y así poder estimularlos con hormona folículo estimulante (FSH) exógena (Cognie et al. 2003), 2) Iniciar el tratamiento de superovulación inmediatamente después de la ovulación (Menchaca y Rubianes 2007), y 3) Asegurar concentraciones altas de progesterona durante la fase inicial del tratamiento superovulatorio (Gonzalez-Bulnes et al. 2004). Pero 
una vez superada la etapa de superovulación, es importante verificar que los embriones generados y recuperados se encuentran en óptimo desarrollo lo que ayudará a disminuir el porcentaje de mortalidad (Cognie et al. 2003).

En rumiantes, se ha demostrado que una dosis única de rBST mejora el desarrollo embrionario si se aplica al momento de la inseminación artificial (Mejía et al. 2012), incrementando el IFNT (vacas lecheras) y aumenta la tasa de concepción (Ribeiro et al. 2014). Asimismo, en programas de ovulación múltiple y transferencia de embriones (OMTE) se ha mejorado la tasa de concepción cuando se aplica la rBST al momento del estro en vacas de leche (Ribeiro et al. 2014), no así en ovejas (Carrera-Chávez et al. 2014). Tanto en vacas como en ovejas se ha mencionado la importancia de iniciar el tratamiento de la rBST para mejorar la tasa de ovulación como el porcentaje de cuates (Safdar y Sadeghi 2016).

Estudios realizados en ovejas y vacas demuestran que la aplicación de la somatotropina bovina recombinante (rBST) previa a la ovulación tiene efectos directos en la fisiología reproductiva, como mayor tamaño del cuerpo lúteo $(\mathrm{CL})$, incremento en la secreción de progesterona y extensión del estro por el alargamiento de la vida media del CL funcional (Navarrete-Sierra et al. 2008, Zarco et al. 2012). También se ha encontrado que altera los componentes del sistema de factores de crecimiento insulínico (IGF), estimulando la esteroidogénesis folicular (Montero-Pardo et al. 2011) y los fluidos uterinos (Costine et al. 2005, Martínez et al. 2011). Adicionalmente, se ha relacionado con el desarrollo de la sensibilidad a la hormona luteinizante $(\mathrm{LH})$ y su mantenimiento en los folículos antrales de ovinos. Por lo que se ha demostrado que la administración de rBST mantiene la sensibilidad de los folículos ováricos a la LH y FSH en ovejas hipofisectomizadas (Eckery et al. 1997). La importancia del presente estudio radicó en aplicar la rBST al inicio del estro, debido a que la mayoría de los estudios que la han utilizado ha sido al iniciar el protocolo de sincronización (Carrera-Chávez et al. 2014) o hasta una semana de iniciar el estro o cuando se inician los tratamientos de superovulación (Mejía et al. 2012). Pero la literatura es escasa en cabras sobre la aplicación de la rBST en diferentes tiempos de un ciclo reproductivo. Por lo anterior, el objetivo fue conocer el efecto de la rBST sobre la actividad ovárica, la cantidad y estadio de los embriones transferibles en cabras al inicio del anestro estacional.

\section{MATERIALES Y MÉTODOS}

\section{Área de estudio}

El estudio se llevó acabo al inicio del anestro estacional (marzo) en el Rancho Cerro de Agua, ubicado en el km 151 de la carretera 57, Galeana, Nuevo León, México ( $24^{\circ} 50^{\prime} 00^{\prime \prime} \mathrm{LN}, 100^{\circ} 04^{\prime} 00^{\prime \prime}$ LO, con altitud de $1600 \mathrm{msnm}$ ). El clima es semiárido, templado con temperatura media anual de $16.1{ }^{\circ} \mathrm{C}$ y precipitación media de 434.5 mm (INEGI 2010).

\section{Manejo y selección de las hembras}

Se utilizaron 30 cabras multíparas de la raza alpina como donadoras de embriones, con la condición de que se tuvieran un parto registrado en la época reproductiva anterior, con un descanso posparto mínimo de cinco meses. Al inicio del experimento, las cabras presentaban un peso vivo de $50.1 \pm 2.3 \mathrm{~kg}$ y condición corporal (CC) de $2.9 \pm$ 0.3 , de acuerdo con la clasificación descrita por Arranz (2002), donde 1 = flaca y 5 = obesa, la CC fue evaluada por dos técnicos expertos en ganado caprino. Las cabras se sometieron 30 días antes del tratamiento hormonal a un manejo sanitario, el cual consistió en la aplicación de vitaminas A, D y E a razón de $1.0 \mathrm{~mL}$ animal $^{-1}$, con una concentración de 500000 U.I, 75000 U.I. y 50 mg, respectivamente (Vigantol ${ }^{\circledR}$ ADE). También se desparasitaron de forma interna con $2.5 \mathrm{mg}$ de Closantel-Panavet ${ }^{\circledR}$ al $5 \%$, vía oral a dosis de $1 \mathrm{~mL}$ por cada $20 \mathrm{~kg}$ de peso vivo. Las cabras permanecieron estabuladas durante el experimento (2 meses) y se alimentaron ad libitum con alfalfa henificada. Adicionalmente se proporcionó un concentrado todos los días a razón de $0.5 \mathrm{~kg}$ animal $^{-1}$ (14\% de proteína cruda y 3.0 Mcal $\mathrm{kg}^{-1}$ de energía metabolizable), mientras que la disponibilidad de agua fue a libre acceso. 


\section{Sincronización y superovulación de las hembras}

La sincronización del estro, se realizó mediante la inserción de dispositivos vaginales con $0.3 \mathrm{~g}$ de progesterona $\left(\mathrm{CIDR}^{\circledR}\right)$ ) durante siete días, el mismo día de la inserción del dispositivo, se aplicó una dosis única de $5 \mathrm{mg}$ de dinoprost trometamina (Lutalyse ${ }^{\circledR}$ ). El tratamiento superovulatorio consistió en la administración de siete dosis de $25 \mathrm{mg}$ de FSHp (Folltropin- $\mathrm{V}^{\circledR}$ ) a partir del cuarto día post-inserción del CIDR y con un intervalo de $12 \mathrm{~h}$ entre cada dosis, para administrar una dosis total de $175 \mathrm{mg}$ de FSHp a cada cabras.

Las cabras fueron bloqueadas de acuerdo al peso, producción de leche y condición corporal, asignandola en forma aleatoria en cada uno de los siguientes tratamientos $T 1=$ grupo testigo $(n=10)$, $\mathrm{T} 2=100 \mathrm{mg}$ de somatotropina bovina recombinante (rBST) (Boostin- ${ }^{\circledR}$, Intervet) $(n=10)$, y T3 $=200 \mathrm{mg}$ de rBST $(n=10)$. En los T2 y T3, la administración de rBST se realizó vía subcutánea después de detectar el estro, desde la remoción de los dispositivos hasta las $72 \mathrm{~h}$ posteriores al retiro; utilizando un macho vasectomizado para la detección del estro. Las cabras se inseminaron vía laparoscopia a un tiempo fijo de $51 \pm 3.4 \mathrm{~h}$ después del retiro del dispositivo con semen congelado importado (BiogenicsLTD), proveniente de sementales de la misma raza, probados genéticamente en cuanto a valores de referencia genética para producción de leche y apreciación lineal (Habilidad de transmisión predicha) y previa prueba de calidad y viabilidad post descongelamiento, relacionados con la concentración espermática y motilidad masal, las cuales fueron evaluadas de acuerdo a la metodología descrita por Evans y Maxwell (1990), dando como referencia un mínimo de 100 millones por pajilla y motilidad mínima de 2.5.

\section{Recolección de embriones}

La recolección de embriones se realizó por la técnica de laparotomía al séptimo día post retiro del dispositivo, mediante la metodología descrita por Baril et al. (1995). Las cabras fueron sometidas a previo ayuno $24 \mathrm{~h}$ antes de la cirugía y se anestesiaron con una mezcla de clorhidrato de ketamina (Anesket ${ }^{\circledR}$ ) a dosis de $20 \mathrm{mg} \mathrm{kg}^{-1}$ de peso vivo y clorhidrato de xilacina (Rompun $\left.{ }^{\circledR}\right)$ a dosis de 0.25 $\mathrm{mg} \mathrm{kg}^{-1}$ de peso vivo. Una vez anestesiada la cabra, se aplicó en el sitio de la incisión abdominal una dosis única de $40 \mathrm{mg}$ de clorhidrato de lidocaína (Pisacaina ${ }^{\circledR} 1 \%$ ). Las cabras se colocaron en posición craneal a $45^{\circ}$ y el aparato reproductor se exteriorizó por una incisión medio-ventral de $6 \mathrm{~cm}$. Posteriormente se utilizó una sonda pediátrica Fouley de balón de $5 \mathrm{~cm}^{3}$ para realizar el lavado de cada uno de los cuernos uterinos aplicando $40 \mathrm{~mL}$ del medio de colección BioLife ${ }^{\mathrm{TM}}$ Advantage Embryo Collection Medium, el cual se mantuvo en baño maría a temperatura de $37^{\circ} \mathrm{C}$. La recolección del medio de cada cuerno uterino se depositó en una caja de Petri (100 x $15 \mathrm{~cm}$ ) para la identificación y clasificación de los embriones mediante un microscopio estereoscopio (Stemi 2000, Carl Zeiss) de acuerdo con los criterios descritos en la guía del Manual del International Embryo Transfer Society (Stringfellow y Seidel 1998).

\section{Variables de estudio}

Se evalúo el intervalo retiro-estro (IRE), contando las horas que pasaron después del retiro del dispositivo hasta la detección del estro. El intervalo retiro-servicio (IRS) se determinó cuantificando las horas que transcurrieron desde el retiro del dispositivo vaginal hasta la inseminación artificial a tiempo fijo. Número de cuerpos lúteos (CL), número de embriones recuperados (ER), número de embriones transferibles (ET), porcentaje de cabras en estro y porcentaje de embriones recuperados, que se calculó en base a los embriones recuperados sobre el número de cuerpos lúteos que se contaron.

\section{Análisis estadístico}

Los datos se analizaron con el paquete estadístico SPSS (Statistical Package for Social Sciences). Todas las variables se compararon con la prueba de Kruskal-Wallis. El porcentaje de cabras en estro y de embriones recuperados, se compararon mediante la prueba de Chi cuadrada. La comparación de medias se realizó por medio de DMS. Las diferencias se consideraron significativas cuando $p<$ 0.05 . 
Tabla 1. Efecto de la rBST en cabras donadoras al final de la época del anestro estacional (medias $\pm E E)$.

\begin{tabular}{ccccc}
\hline & \multicolumn{4}{c}{ Somatotropina bovina recombinante (rBST) } \\
Variable & T1 $=$ Control & T2 $=100 \mathrm{mg}$ & T3 $=200 \mathrm{mg}$ & Probabilidad \\
\hline Número de hembras & 10 & 10 & 10 & \\
Cabras en estro (\%) & $80.0(10 / 8)$ & $100.0(10 / 10)$ & $100.0(10 / 10)$ & 0.212 \\
Intervalo retiro-estro $(\mathrm{h})$ & $28.8 \pm 0.8$ & $28.9 \pm 2.4$ & $27.4 \pm 1.0$ & 0.972 \\
Intervalo retiro-servicio (h) & $51.3 \pm 0.1$ & $51.7 \pm 0.6$ & $51.1 \pm 0.1$ & 0.722 \\
Cuerpos lúteos $(\mathrm{n})$ & $3.2 \pm 2.4^{b}$ & $5.3 \pm 2.1^{a}$ & $7.0 \pm 2.8^{a}$ & 0.002 \\
Embriones recuperados (n) & $3.2 \pm 2.1^{c}$ & $4.5 \pm 2.5^{b}$ & $6.0 \pm 4.9^{a}$ & 0.001 \\
Embriones recuperados (\%) & $100.0(32 / 32)^{a}$ & $84.9(45 / 53)^{b}$ & $85.7(60 / 70)^{b}$ & 0.02 \\
Mórulas (n) & $3.0 \pm 0.3^{a}$ & $2.0 \pm 0.4^{a}$ & $1.0 \pm 0.2^{b}$ & 0.008 \\
Blastocitos (n) & $0.0 \mathrm{c}$ & $3.0 \pm 0.7^{b}$ & $6.0 \pm 0.4^{a}$ & 0.001 \\
\hline Valores con diferente superíndice son estadísticamente diferentes $(\mathrm{p}<0.05)$.
\end{tabular}

\section{RESULTADOS Y DISCUSIÓN}

Para las variables de la sincronización del estro como el porcentaje de cabras en estro, el IRE, IRS no se presentaron diferencias significativas entre los tratamientos evaluados ( $p>0.05$, Tabla 1$)$. Para el número de cuerpos lúteos, se presentó diferencia significativa $(p=0.002)$ entre los tratamientos, siendo mayor para el T3. Para el número y porcentaje de embriones recuperados se presentaron diferencias significativas ( $p=0.001, p=0.02)$ entre tratamientos, siendo mayor en ambos en T3. Para el total de mórulas fue mayor en $T 1$, y para blastocitos recuperados fue mayor en T3 que T2 ( $p=0.0008$ y $p=0.001)$ respectivamente; siendo en el caso que $\mathrm{T} 1$ no se recolectó ningún blastocito.

En general, se incrementó de forma significativa la producción de embriones transferibles en cabras con la aplicación de la rBST al momento de iniciado el estro y durante el inicio del anestro estacional. El comportamiento favorable de los tratamientos de rBST, sobre el porcentaje de embriones recuperados y su estadio, pudo deberse al aumento en las primeras $24 \mathrm{~h}$ de aplicación de IGF-I (Safdar y Sadeghi 2016) y disminuciones del INFT, ejerciendo un efecto estimulador en la proliferación y mitogénesis de células tecales y de la granulosa, favoreciendo la formación de folículos preovulatorios como se reporta en otras especies de mamíferos, como la yegua (Equus ferus caballus) (Davidson et al. 2002); o bien favoreciendo de forma directa el incremento en la población de folículos preantrales (Moreira et al. 2002b). Pero hoy en día se tiene poca información con respecto a este evento en cabras.

En este estudio, el efecto de rBST también se vio reflejado en aumento del número de $\mathrm{CL}$, lo que coincide con lo reportado por trabajos en ovejas (Zarco et al. 2012, Montero-Pardo et al. 2011), quienes obtuvieron hasta 4.5 más de CL cuando se aplicó rBST. En el presente trabajo, la condición corporal se manejó de forma adecuada desde el inicio del trabajo (2.9) para que no fuera un factor determinante en la respuesta de la dosis de rBST (100 y 200 $\mathrm{mg}$ ) que se aplicó a las cabra al inicio del estro, ya que de lo contrario al ser sometidas a un programa de transferencia de embriones, se puede afectar el desarrollo folicular y de embriones a mejores estadios (morula y blastocisto), más aún si el tiempo de aplicación de la rBST se realiza posterior al estro. Al respecto Moreira et al. (2002a) en vacas lecheras, encontraron diferencias en el número de folículos desarrollados y por lo tanto de cuerpos lúteos, cuando se aplicó la rBST en la inseminación y con diferente condición corporal. En este trabajo se logró impactar sobre el desarrollo de blastocitos aumentando el número de los mismos, para las cabras que recibieron $100 \mathrm{mg}$ y $200 \mathrm{mg}$ de rBST, las cuales son iguales a los reportados por Safdar y Sadeghi (2016), en ovejas. Esto se soporta por otras investigaciones realizadas in vitro, por el hecho de que embriones de dos células expresan más temprano el mRNA para los receptores de la hormona del crecimiento (Baldassarre 2007) y el adicionar rBST en el medio de cultivo acelera el desarrollo embrionario (Costine et al. 2005). Sin embargo, debe continuarse con líneas de investigación, donde se evalúe el efecto de dosis 
mayores de $100 \mathrm{mg}$ de rBST, sobre el desarrollo embrionario, ya que se ha reportado en otros estudios que al exponer los embriones a altas concentraciones de Insulina e IGF-1, sufren apoptosis y por lo tanto al ser transferidos se reabsorberán, lo que afecta la tasa de gestación (Chi et al. 2000).
Se encontró efecto benéfico de la rBST sobre la actividad ovárica y cantidad de embriones obtenidos dependiendo de la dosis utilizada. Sin embargo, se debe de considerar evaluar la acción directa de la rBST sobre las hormonas que actúan directamente sobre el crecimiento y desarrollo folicular de las cabras y su efecto sobre el desarrollo embrionario.

\section{LITERATURA CITADA}

Arranz LO (2002) Guía práctica de ganado ovino de leche. INEA-EXCMA. España. 34p.

Baldassarre H (2007) Assisted reproduction in goats: artificial insemination to cloning. Revista Brasileña de Reproducción Animal 31: 274-282.

Baril G, Brebion P, Chesné P (1995) Manual de formación práctica para el transplante de embriones en ovejas y cabras. FAO. Italia. 175p.

Carrera-Chávez JM, Hernández-Cerón J, López-Carlos MA, Lozano-Domínguez RR, Molinar F, EchavarríaCháirez FG, et al. (2014) Superovulatory response and embryo development in ewes treated with two doses of bovine somatotropin. Animal Reproduction Science 151: 105-111.

Chi MM-Y, Schlein AL, Moley KH (2000) High insulin-like growth factor 1 (IGF-1) and insulin concentration trigger apoptosis in the mouse blastocyst via down-regulation of the IGF-1 receptor. Endocrinology 141: 47844792.

Cognie Y, Baril G, Poulin N, Mermillod P (2003) Current status of embryo technologies in sheep and goat. Theriogenology 59: 171-188.

Costine BA, Inskeep EK, Wilson ME (2005) Growth hormone at breeding modifies conceptus development and postnatal growth in sheep. Journal of Animal Science 83: 810-815.

Davidson RT, Chamberlain S, Bridges S, Spicer J (2002) Effect of follicle size on in vitro production of steroids and insulin-like growth factor IGF-I, IGF-II and the IGF-binding proteins by equine ovarian granulosa cells. Biology of Reproduction 66: 1640-1648.

Eckery DC, Moeller CL, Nett TM, Sawyer HR (1997) Localization and quantification of binding sites for folliclestimulating hormone, luteinizing hormone, growth hormone, and insulin-like growth factor I in sheep ovarian follicles. Biology of Reproduction 57: 507-513.

Evans G, Maxwell WMC (1990) Inseminación artificial de ovejas y cabras. Editorial Acribia, Zaragoza, España. 204p.

Gonzalez-Bulnes A, Baird DT, Campbell BK, Cocero MJ, Garcia-Garcia RM, Inskeep EK, et al. (2004) Multiple factors affecting the efficiency of multiple ovulation and embryo transfer in sheep and goats. Reproduction Fertility and Development 16: 421-435.

INEGI (2010) Estadísticas históricas meteorológicas en el municipio de Galeana, Nuevo León. Instituto Nacional de Estadística y Geografía. www.inegi.org.mx/est/contenidos/espanol/sistemas/cem05/info/nln/.../c19017_ 01.xls. Fecha de consulta: 24 de abril de 2018. 
Martínez Aguilar M, Gutiérrez CG, Domínguez Hernández YM, Hernández Cerón J (2011) Respuesta estral y tasa de preñez en cabras en anestro estacional tratadas con progestágenos y somatotropina bovina. Revista Mexicana de Ciencias Pecuarias 2: 221-227.

Mejía O, Palma-Irizarry M, Rosas J, Madrid-Marina V, Valencia MJ, Zarco L (2012) Administration of recombinant bovine somatotropin (rbST) at the time of breeding in superovulated fertile and subfertile ewes. Small Ruminant Research 102: 51-56.

Menchaca A, Rubianes E (2007) Pregnancy rate obtained with short-term protocol for timed artificial insemination in goats. Reproduction in Domestic Animals 42: 590-593.

Montero-Pardo A, Hernández-Cerón J, Rojas-Maya S, Valencia J, Rodríguez-Cortez A, Gutiérrez CG (2011) Increased cleavage and blastocyst rate in ewes treated with bovine somatotropin 5 days before the end of progestin-based estrous synchronization. Animal Reproduction Science 125: 69-73.

Moreira F, Badinga L, Burnley C, Thatcher WW (2002a) Bovine somatotropin increases embryonic development in superovulated cows and improves post-transfer pregnancy rates when given to lactating recipient cows. Theriogenology 57: 1371-1387.

Moreira F, Paula-Lopes FF, Hansen PJ, Badinga L, Thatcher WW (2002b) Effects of growth hormone and insulinlike growth factor-I on development of in vitro derived bovine embryos. Theriogenology 57: 895-907.

Navarrete-Sierra LF, Cruz-Tamayo AA, González-Parra El, Piña-Aguilar RE, Sangines-García JR, Toledo-López $V$, et al. (2008) Effect of recombinant growth hormone (rBST) application on superovulatory response and embryo viability in hair ewes. Revista Científica, Facultad de Ciencias Veterinarias de la Universidad del Zulia 18: 175-179.

Ribeiro ES, Bruno RGS, Farias AM, Hernández-Rivera JA, Gomes GC, Surjus R, et al. (2014) Low doses of bovine somatotropin enhance conceptus development and fertility in lactating dairy cows. Biology of Reproduction 90: 1-12

Safdar AHA, Sadeghi AA (2016) Superovulatory response and embryonic progressive in Iranian Qezel ewes treated with two different concentrations of bovine somatotropin. Asian Pacific Journal of Reproduction 5: 221-226.

Stringfellow D, Seidel SM (1998) Manual of the International Embryo Transfer Society. 3rd ed. IETS, Savoy, IL, EUA. 170p.

Zarco L, Mejía O, Palma-Irizarryb M, Rosasd J, Madrid-Marinac V, Valenciad MJ (2012) Administration of recombinant bovine somatotropin (rBST) at the time of breeding in superovulated fertile and subfertile ewes. Small Ruminant Research 102: 51-56. 